between $61.3 \%$ and 98.1\%. Procalcitonin-based protocols decreased antibiotic prescription (RR 0.56 [0.43, 0.73]) and the total antibiotic exposure (MD $-3.83[-4.32,-3.35])$, without affecting clinical outcomes such as rate of treatment failure (RR $0.81[0.62,1.06])$, length of hospitalisation (MD $-0.76[-1.95$, $0.43]$ ), exacerbation recurrence rate (RR $0.96[0.69,1.35])$ or mortality (RR 0.99 [0.57, 1.70]). However, the quality of the available evidence is low to moderate because of methodological limitations and small overall study population.

Conclusion Procalcitonin-based protocols to guide the administration of antibiotics in patients presenting with AECOPD appear safe and clinically effective. The quality of the available evidence is low-to-moderate because of methodological limitations and small overall population. Thus, additional appropriately designed and powered confirmatory randomised controlled trials are required.

\section{P52 COPD AND PERIODONTITIS: CO-MORBIDITY YES OR NO?}

${ }^{1} S$ Hobbins, ${ }^{1} \mathrm{~A}$ Usher, ${ }^{2} \mathrm{~S}$ Parmar, ${ }^{1} \mathrm{R}$ Stockley. ${ }^{1}$ Queen Elizabeth Hospital, Birmingham, UK; ${ }^{2}$ School of Dentistry, University of Birmingham, Birmingham, UK

10.1136/thoraxjnl-2016-209333.195

Introduction Chronic obstructive pulmonary disease (COPD) is an inflammatory disease associated with comorbidities including periodontitis. ${ }^{1-2}$ Periodontitis is characterised by plaque build-up, anaerobic bacterial overgrowth and gingival inflammation which promotes recruitment and activation of neutrophils leading to alveolar bone destruction and tooth loss. However, the characterisation of periodontitis varies between studies causing some uncertainty of any association.

Aim To determine whether clinical indices of periodontitis affects its prevalence in COPD patients with and without Alpha-1-antitrypsin deficiency (AATD) and any association with lung function.

Methods 108 COPD and 63 PiZ AATD patients underwent dental examinations and lung function testing as part of an EU FP7 cross sectional study.

Varying definitions of periodontitis used in previous publications were applied; including criteria from the Centres for Disease Control and Prevention in collaboration with the American Academy of Periodontology CDC-AAP (CDC-AAP) and 5th European Workshop in Periodontology.

Periodontal indices of probing depth (PD - depth from gingival margin to the base of periodontal pocket) and clinical attachment level (CAL - distance from the cemento-enamel junction to the gingival margin plus probing depth) were then compared to lung function parameters.

Results The prevalence of periodontitis varied depending on the definition used.

Prevalence ranged from $0.7-98.6 \%$ for the whole cohort, with the lowest prevalence for average probing depth $>4 \mathrm{~mm}$, but CDC-AAP criteria gave a prevalence of $84.2 \%$ and $98.6 \%$ with the 5 th European workshop criteria.

Lung function was significantly correlated with indices of periodontitis for AATD patients; see Table.

Conclusions The prevalence of periodontitis depends on the definition used. PD is a marker of current status, whilst CAL represents cumulative disease activity, rather like current lung function parameters.
Abstract P52 Table 1 Relationships between clinical indices of periodontitis and lung function in COPD and AATD

\begin{tabular}{|c|c|c|c|c|}
\hline & \multicolumn{4}{|c|}{ Spearman's Rho and p-value } \\
\hline & \multicolumn{2}{|c|}{ Average Probing Depth (PD) } & \multicolumn{2}{|c|}{$\begin{array}{l}\text { Average Clinical Attachment } \\
\text { Level (CAL) }\end{array}$} \\
\hline & COPD & AATD & COPD & AATD \\
\hline \multirow[t]{2}{*}{$\%$ predicted FEV1 } & 0.085 & -0.42 & -0.03 & -0.52 \\
\hline & $p=N S$ & $p<0.01$ & $p=N S$ & $p<0.001$ \\
\hline \multirow[t]{2}{*}{$\%$ predicted TLCO } & -0.06 & -0.34 & 0.01 & -0.51 \\
\hline & $p=N S$ & $p<0.01$ & $\mathrm{p}=\mathrm{NS}$ & $p<0.001$ \\
\hline \multirow[t]{2}{*}{$\%$ predicted KCO } & -0.10 & -0.30 & -0.04 & -0.42 \\
\hline & $\mathrm{p}=\mathrm{NS}$ & $p<0.05$ & $p=N S$ & $p<0.01$ \\
\hline
\end{tabular}

Periodontal indices are correlated with lung function parameters in AATD patients which could reflect the inflammatory and predominantly neutrophilic pathophysiology leading to excessive tissue destruction in both diseases.

\section{REFERENCES}

1 Shen TC, et al. Risk of Periodontal Diseases in Patients With Chronic Obstructive Pulmonary Disease: A Nationwide Population-based Cohort Study. Medicine (Baltimore) 2015; 94(46):e2047.

2 Chung JH, et al. J Periodontol 2016:1-11.

\section{P53 PREDICTING POOR OUTCOMES IN COPD PATIENTS DEEMED 'LOW RISK' BY DOSE SCORE}

${ }^{1}$ LA Rigge, ${ }^{2} \mathrm{NA}$ Coombs, ${ }^{1} \mathrm{M}$ Johnson, ${ }^{3} \mathrm{D}$ Culliford, ${ }^{4} \mathrm{~L}$ Josephs, ${ }^{5} \mathrm{~N}$ Williams, ${ }^{4} \mathrm{M}$ Thomas, ${ }^{1} \mathrm{~T}$ Wilkinson. ${ }^{1} \mathrm{NIHR}$ CLAHRC Wessex, University of Southampton, Clinical and Experimental Sciences and University Hospitals Southampton Foundation Trust, Southampton, UK; ${ }^{2}$ University of Southampton, Primary Care and Population Sciences, Southampton, UK; ${ }^{3}$ NIHR CLAHRC Wessex, Methodological Hub, Southampton, UK; ${ }^{4}$ NIHR CLAHRC Wessex, University of Southampton, Primary Care and Population Sciences, Southampton, UK; ${ }^{5}$ University of Southampton, Clinical and Experimental Sciences and University Hospitals Southampton Foundation Trust, Southampton, UK

\subsection{6/thoraxjnl-2016-209333.196}

Introduction COPD continues to cause a substantial symptom, mortality and financial burden in the UK. Current treatment strategies are predominantly reactive as insufficient evidence exists to successfully target clinical resource into pre-emptive 'early interventions'. The DOSE (dyspnoea, obstruction, smoking status and exacerbation) score has been validated as a risk predictor for mortality, hospitalisation and poorer health status. However, only a small proportion of COPD patients with poor outcomes have high DOSE scores. We sought to establish if clinical characteristics can be used to pre-emptively identify those COPD patients vulnerable to future poor health status by using an electronic database of anonymised patient records-the Hampshire Health Record Analytical Database (HHRA).

Methods Within our HHRA database COPD cohort, we identified a cohort of 6890 patients who fell into the 'low risk' category by DOSE score $(<4)$. Within this group, a subset met the criteria for poor COPD outcomes over the next four years, defined as; death (all cause), COPD related hospital admission, a DOSE score increase of $\geq 2$ points or a subsequent DOSE score of $\geq 4$ (high risk). We used logistic regression analysis to examine the association between demographic and clinical characteristics documented by Read code at baseline and those who subsequently fell into the poor outcomes subgroup. 